\title{
An Electrically Small Antenna in Package Design with Embedded Electronics for RPW Detection
}

\author{
Haoran Zhang and Atif Shamim \\ Electrical Engineering Program, CEMSE Division \\ King Abdullah University of Science and Technology, Thuwal, Saudi Arabia \\ Haoran.zhang@kaust.edu.sa; Atif.shamim@kaust.edu.sa
}

\begin{abstract}
The tracking and detection of Red Palm Weevils (RPWs) are highly desirable for efficient pest control. Bluetooth Low Energy (BLE) tracking system is a good option for real-time monitoring, however, for mounting the system on the insect, it must be miniaturized, particularly the antenna part. In this paper, we propose an electrically small inverted $F$ antenna which is realized on the BLE system package, qualifying it as an antenna in package (AiP) design. The antenna has been designed with the embedded electronics and has a highly compact size of $0.05 \lambda \times$ $0.05 \lambda \times 0.025 \lambda$. It is very well matched at $2.48 \mathrm{GHz}$ with $17 \mathrm{MHz}$ bandwidth. It demonstrates a maximum detection range of $\mathbf{3 0 0}$ meters.
\end{abstract}

\section{Keywords-RPW; BLE; inverted F antenna; AiP}

\section{INTRODUCTION}

RPWs represent one of the most debilitating pests for palm trees all over the world, due to their destructive behavior and rapid reproduction. Hence, detailed information on the biological behavior and dynamics of RPW is needed to support risk assessment of identified infestations such as dispersal ranges. In order to monitor RPW, tracking system needs to be attached or mounted on the RPW's body. However, the available area on RPW's back is only around $8 \mathrm{~mm} \times 8 \mathrm{~mm}$ for the placement of the tracking system. The overall tracking system should be lightweight and small so that it will not affect the insect's normal movement and behavior. The very small available space makes it a challenging design and thus the tracking system, especially the antenna part, must be comprehensively miniaturized without losing much on the performance.

Several approaches have been reported in the previous work for insect tracking. Harmonic transponders with long wire antennas were attached to insects' back for tracking [1, 2]. They successfully achieved insect detection over long ranges with high power transmitters and receivers. However, there is no identification module integrated, meaning that they cannot distinguish between different insects. In [3], active radio transmitters were proposed for insect tracking and achieved good read ranges, but they require long wire antennas and the use of batteries. In order to avoid using a relatively heavy battery, passive RFID was selected for tracking insects [4]. But the antenna size is also considerably large due to the low operation frequency range of passive RFID. Thus, it can be concluded that a compact antenna design is highly desirable for insect tracking system. BLE system has good potential for RPW tracking due to its properties of small size, lightweight, and low power consumption. This paper proposes a compact inverted $\mathrm{F}$ antenna design for RPW detection by BLE system. The antenna has been designed with embedded electronics and can achieve a detection/read range of 300 meters.

\section{SYSTEM LEVEL DESIGN}

The proposed design is an active BLE based tracking system, which comprises of BLE active and passive components mounted on a printed circuit board (PCB), battery, and BLE antenna. This paper mainly focuses on the compact BLE antenna design. Antenna's performance can be strongly affected by the final package structure, especially due to the presence of the PCB and the battery. Therefore, the entire system with packaging has been modeled in HFSS, as shown in Fig. 1. The two main parts of the system are the PCB package and the battery holder. The battery holder is used for a small button battery. The PCB is attached on top of the battery holder, while the DC connection between the battery and the PCB is realized through metal traces printed on the battery holder. The PCB package provides protection for the BLE circuits and also acts as the substrate for the antenna. All the metal traces, vias, and chip models are included to ensure reliable simulations.

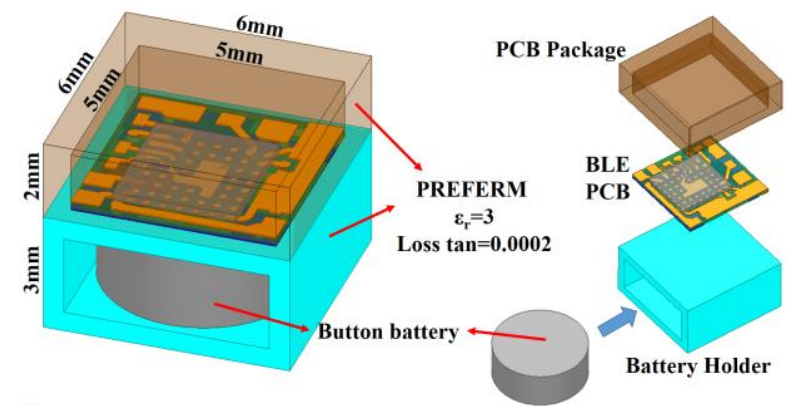

Fig. 1. Geometry of the BLE system package and its enlarged view

\section{Antenna On PACKAgE DESIGN \& OPTIMIZATION}

Inverted $\mathrm{F}$ antenna is suitable for this design because it is shorter and more compact than other wire antennas, such as monopole. In addition, the impedance matching of inverted $\mathrm{F}$ antenna can be controlled without adding any extra matching component, which can considerably increase the PCB size in such a compact system. The inverted F type AiP design is shown in Fig. 2. The copper traces (green), including the antenna as well as the short and back lines, are realized on the outer surfaces of the PCB package, while most area of the battery holder's inner and outer surfaces are covered with copper ground (orange) to ensure that antenna has a solid ground. In order to minimize the radiation cancellation, the antenna line is meandered in such a way that the currents on the two close-by metal traces have the 
same direction. In addition, the lumped port is placed between the RF and ground pins of the BLE chip (nRF52832).

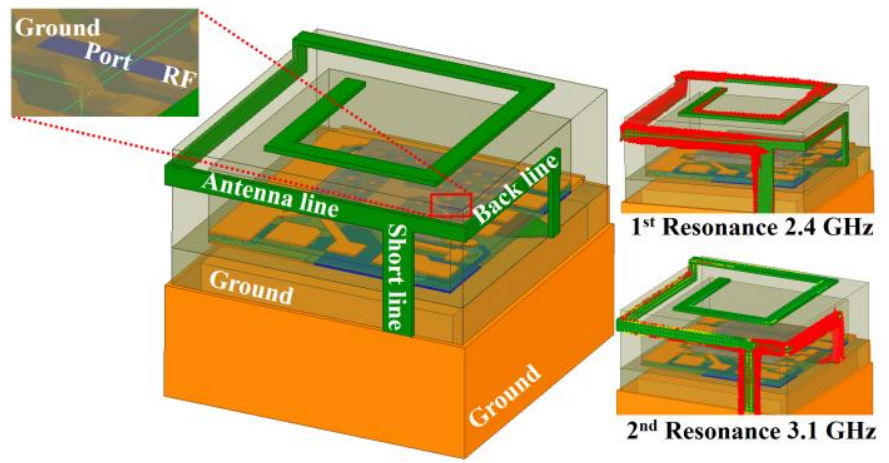

Fig. 2. Geometry of the BLE antenna in package and its port excitation

The initial simulation results show that there are two resonances which are around $2.4 \mathrm{GHz}$ and $3.1 \mathrm{GHz}$, respectively. The currents of the first resonance mainly flow on the antenna line and the short line, while the second one is concentrated on the short line and the back line. The current distributions indicate the critical areas for the two resonances (shown in Fig. 2).

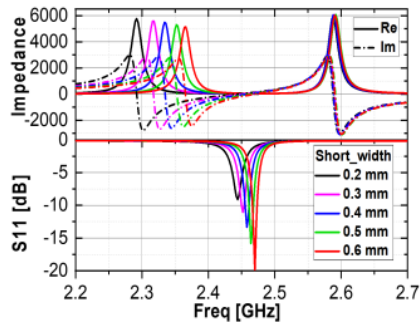

(a)

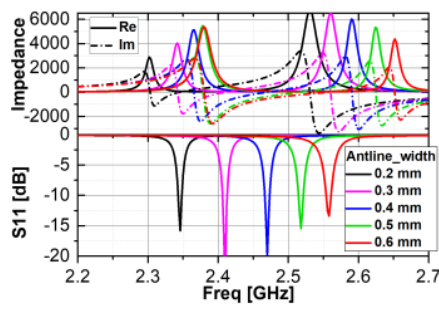

(c)

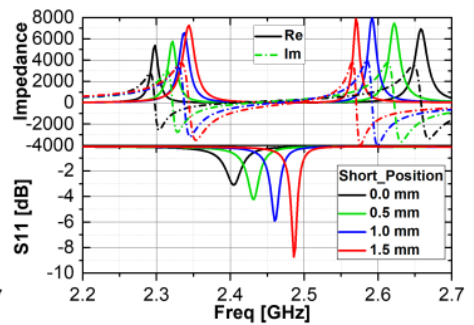

(b)

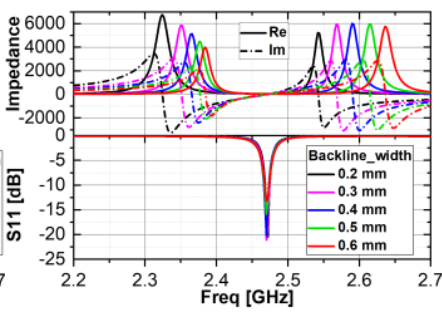

(d)
Fig. 3. Parametric simulation (a) short line width, (b) short line position, (c) antenna line width, (d) back line width

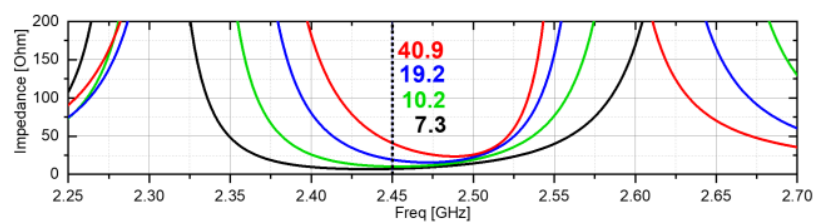

Fig. 4. Real part of antenna impedance from Fig. 3(b)

In order to match antenna impedance and further investigate the influences of the antenna dimensions on the resonances, four parametric studies have been done, as shown in Fig. 3. It can be seen from Fig. 3(a) that a wider short line leads to better impedance matching and higher resonance frequency. Moreover, it mainly affects the first resonance. As shown in Fig. 3(b), when the short line position is closer to the feeding point, the first resonance gets better impedance matching and shifts to a higher frequency because the electrical length of the antenna line gets longer. Fig. 3(c) shows that the wider antenna line results in a higher frequency of first resonance. Fig. 3(d) indicates that the back line width does not have much impact on the first resonance, because most of the current related to the first resonance is distributed on the antenna line instead of the back line. As the two resonance frequencies get close to each other, a higher antenna input resistance can be obtained for better impedance matching, as shown in Fig. 4.

After optimization, the widths of short line, antenna line, and back line are chosen to be $0.6 \mathrm{~mm}, 0.4 \mathrm{~mm}$, and $0.3 \mathrm{~mm}$ respectively. The simulated reflection coefficient and radiation pattern are shown in Fig. 5. It can be seen that a bandwidth of $17 \mathrm{MHz}$ is obtained within the BLE frequency range. Though it's a narrow band, it's sufficient to cover the $2480 \mathrm{MHz}$ advertising channel for establishing communication. In addition, the antenna presents a good donut-shaped radiation pattern, indicating a good angle coverage. The antenna achieves an acceptable maximum gain of $-4 \mathrm{dBi}$, which is acceptable considering the highly compact size of $0.05 \lambda \times 0.05 \lambda \times 0.025 \lambda$ with an electrical size of $k a=0.23$. When the same BLE chip is used in the receiver and connected to an isotropic antenna, a maximum detection range of $300 \mathrm{~m}$ can be achieved.

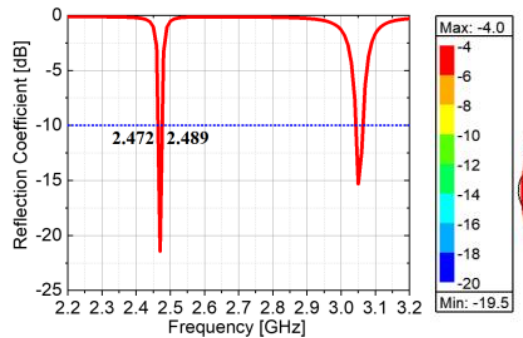

(a)

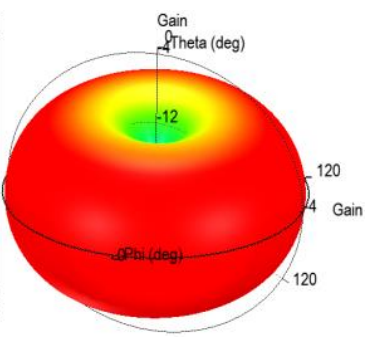

(b)
Fig. 5. Simulated results (a) reflection coefficient, (b) radiation pattern

\section{CONCLUSION}

A highly compact AiP design of inverted $\mathrm{F}$ antenna in a BLE system has been developed for RPW detection. A meandering antenna wire on the outer surfaces of PCB package is proposed to miniaturize the antenna size. The proposed antenna achieves $17 \mathrm{MHz}$ bandwidth in BLE frequency range, a maximum gain of $-4 \mathrm{dBi}$, and a maximum detection range of $300 \mathrm{~m}$, which are sufficient to be used in the RPW tracking system.

\section{REFERENCES}

[1] B. G. Colpitts and G. Boiteau, "Harmonic radar transceiver design: miniature tags for insect tracking," IEEE Transactions on Antennas and Propagation, vol. 52, no. 11, pp. 2825-2832, 2004.

[2] N. Tahir and G. Brooker, "Toward the Development of Millimeter Wave Harmonic Sensors for Tracking Small Insects," IEEE Sensors Journal, vol. 15, no. 10, pp. 5669-5676, 2015.

[3] R. Hamidi, P. Couzi, K. Khfif, and D. Rochat, "Impact of active and passive radio tags on the flying and burrowing behavior of the red palm weevil, Rhynchophorus ferrugineus," Applied Entomology and Zoology, vol. 52, no. 1, pp. 165-173, 2017/02/01 2017.

[4] S. Särkkä, V. Viikari, and K. Jaakkola, "RFID-based butterfly location sensing system," in 2014 22nd EUSIPCO, 1-5 Sept. 2014, pp. 2045-204 